\title{
Identification of high-risk patients by human epididymis protein 4 levels during follow-up of ovarian cancer
}

\author{
KARINA DAHL STEFFENSEN ${ }^{1,2}$, MARIANNE WALDSTR $\emptyset^{3}{ }^{3}$, IVAN BRANDSLUND ${ }^{4}$, \\ BENTE LUND ${ }^{5}$, SARAH MEJER SØRENSEN ${ }^{6}$, MAX PETZOLD $^{7}$ and ANDERS JAKOBSEN ${ }^{1,2}$ \\ ${ }^{1}$ Department of Oncology, Vejle Hospital, DK-7100 Vejle; ${ }^{2}$ Institute of Regional Health Research, University of \\ Southern Denmark, DK-5000 Odense; Departments of ${ }^{3}$ Pathology and ${ }^{4}$ Clinical Biochemistry, Vejle Hospital, DK-7100 Vejle; \\ ${ }^{5}$ Department of Oncology, Aalborg University Hospital, DK-9100 Aalborg; ${ }^{6}$ Faculty of Health Sciences, \\ University of Copenhagen, DK-2200 Copenhagen, Denmark; ${ }^{7}$ Centre for Applied Biostatistics, \\ Sahlgrenska Academy, University of Gothenburg, S-405 30 Gothenburg, Sweden
}

Received February 16, 2016; Accepted April 13, 2016

DOI: $10.3892 / \mathrm{ol} .2016 .4533$

\begin{abstract}
The majority of ovarian cancer patients with advanced disease at diagnosis will relapse following primary treatment, with a dismal prognosis. Monitoring the levels of serum markers in patients under follow-up may be essential for the early detection of relapse, and for distinguishing high-risk patients from those with less aggressive disease. The aim of the present study was to investigate the possible predictive value of human epididymis protein 4 (HE4) and carbohydrate antigen 125 (CA125) in relation to recurrence of epithelial ovarian cancer by measuring the two markers during follow-up subsequent to surgery and adjuvant first-line carboplatin/paclitaxel chemotherapy. Serum HE4 and CA125 were analyzed in 88 epithelial ovarian cancer patients at the end of treatment and consecutively during follow-up. The patients were divided into a high-risk and a low-risk group based on having an increase in HE4 and CA125 levels above or below 50\% during follow-up, relative to the baseline (end-of-treatment) level. Disease recurrence was detected in 55 patients during follow-up. Patients with an increase in $\mathrm{HE} 4$ of $>50 \%$ at 3-and 6-month follow-up compared to the end-of-treatment sample had significantly poorer progression-free survival (PFS) [hazard ratio (HR), 2.82 (95\% CI, 0.91-8.79; P=0.0052) and HR, 7.71 (95\% CI, 3.03-19.58; $\mathrm{P}<0.0001)$, respectively]. The corresponding 3- and 6-month biomarker assessments for increased CA125 levels (>50\%) showed HRs of 1.86 (95\% CI, 0.90-3.80; $\mathrm{P}=0.0512)$ and 2.55 (95\% CI, 1.39-4.68; $\mathrm{P}=0.0011)$, respectively. Multivariate analysis confirmed HE4 as a predictor of short PFS, with an HR of 8.23 (95\% CI, 3.28-20.9; P<0.0001) at 6-month follow-up. The increase of CA125 was not a significant prognostic factor
\end{abstract}

Correspondence to: Dr Karina Dahl Steffensen, Department of Oncology, Vejle Hospital, Kabbeltoft 25, DK-7100 Vejle, Denmark E-mail: karina.dahl.steffensen@rsyd.dk

Key words: ovarian cancer, human epididymis protein 4, carbohydrate antigen 125 , follow-up, recurrence, biomarker in multivariate analysis for PFS. In conclusion, HE4 appears to be a sensitive marker of recurrence and instrumental in risk assessment during the first 6 months of follow-up.

\section{Introduction}

The majority of patients with advanced ovarian cancer will experience disease recurrence within a few years from the time of diagnosis (1). Due to the nature of the disease, with its location in the small pelvis and spread in the form of diffuse carcinosis, there are no reliable methods of detecting early recurrence using ultrasound, computed tomography (CT) or magnetic resonance imaging $(2,3)$. Furthermore, it is well known that serum levels of carbohydrate antigen 125 (CA125) have a considerable lead-time before clinically detectable recurrence $(4,5)$.

Currently, the clinical consequence of rising CA125 levels remains an issue of great debate, particularly as to whether re-treatment should be initiated based only on biochemical CA125 recurrence. Rustin et al (6) demonstrated that survival time was not improved when treatment was based on biochemical recurrence alone. However, it should be noted that this study investigated only women who experienced normalization of CA125 during their first-line treatment. Furthermore, the study has been criticized for its very diverse relapse treatment, including different chemotherapy regimens, which may not have been in accordance with the current standard. Also, none of the patients were offered secondary cytoreductive surgery that may have led to improved survival; this issue is currently under intense discussion in the scientific community, and the results of the ongoing randomized DESKTOP III (7) study are eagerly awaited to clarify whether surgery for relapse will improve the outcome for patients with recurrent disease. It may be highly relevant to practice active monitoring of women after the end of treatment to detect recurrence as early as possible, particularly if the DESKTOP III study demonstrates a survival benefit. Furthermore, a retrospective study by Fleming et al (8) indicated that each week delay of treatment following the first CA125 elevation in recurrent ovarian cancer correlated with a $3 \%$ increased chance of suboptimal resection at secondary 
cytoreductive surgery, and therefore CA125 surveillance increased optimal resectability at secondary cytoreductive surgery. Thus, the current situation calls for better methods for early detection of recurrence with the perspective of curatively intended surgical and/or non-surgical treatment. CA125 is insufficient for a number of reasons, including the fact that it is not always elevated in patients with mucinous tumors (9).

Human epididymis protein 4 (HE4) is a relatively new biomarker approved by the United States Food and Drug Administration (FDA) for monitoring of patients with epithelial ovarian cancer. HE4 is encoded by the WFDC2 gene located on chromosome 20q12-13.1 (10) and belongs to the family of whey-acidic four-disulfide core proteins with suspected trypsin-inhibitor properties (11). However, the biological role of HE4 has not yet been identified (12). HE4 is upregulated in ovarian cancer compared to other types of carcinomas and benign ovarian tumors $(13,14)$. Recent studies have identified HE4 as a complementary marker for ovarian cancer that can be elevated in some cases where CA125 is not (15-21); however, its potential value in the early detection of recurrence has not been elucidated.

The aim of the present study was to explore the clinical value of serial measurements of HE4 and CA125 during follow-up for the early detection of recurrence, and the additive value of combining the two markers.

\section{Materials and methods}

Study population. The current study included patients with ovarian cancer who had completed first-line combination chemotherapy with paclitaxel $\left(175 \mathrm{mg} / \mathrm{m}^{2}\right.$ intravenously) and carboplatin $(\mathrm{AUC}=5)$ every 3 weeks at two Danish Hospitals (Aalborg University Hospital, Aalborg, Denmark; and Vejle Hospital, Vejle, Demark) and attended follow-up according to national guidelines. Patients with a serum sample drawn at the end of chemotherapy and $\geq 2$ post-chemotherapy blood samples were included in the study.

All patients were entered as part of a translational research protocol, with peripheral venous blood samples drawn at the end of chemotherapy and at every scheduled follow-up visit: Every 3 months for the first two years, every 6 months for the third year, and once a year for the fourth and fifth years. Clinical data were recorded in detailed case report forms. Detailed patient characteristics have been given elsewhere (22).

The study was carried out in compliance with the Helsinki II Declaration (23) and all patients signed an informed consent form. The Danish Biomedical Research Ethics Committee and the Danish Data Protection Agency approved the study according to Danish law. Recurrence of disease was defined according to the Gynecological Cancer Intergroup CA125 criteria $(24,25)$ and/or radiological confirmation of tumor recurrence, whichever occurred first. Biochemical recurrence detected by CA125 required CT confirmation to verify the diagnosis of recurrence.

Serum CA125 assay. The quantitative levels of serum CA125 were determined using the commercially available CanAg CA125 Enzyme Immunometric Assay (EIA) kit (cat. no.,400-10; Fujirebio Diagnostics AB, Gothenburg, Sweden) with inter- and intra-assay coefficients of variation $(\mathrm{CV})$ of $\leq 10 \%$ and a sensitivity of $1.5 \mathrm{IU} / \mathrm{ml}$. The assay is based on a direct sandwich technique using two mouse monoclonal antibodies, Ov197 and Ov185, directed against two independent epitopes of the protein core of the CA125 antigen. The analysis was performed in $25 \mu 1$ of serum and followed the manufacturer's protocol.

Serum HE4 assay. HE4 serum levels were determined by the enzyme-linked immunosorbent assay technique using a commercially available FDA-approved kit (HE4 EIA kit, cat. no., 404-10; Fujirebio Diagnostics AB). The analysis used $25 \mu 1$ serum; the analytical steps were conducted according to the manufacturer's instructions and have been described in further detail in a previous publication from our group (21).

HE 4 control 1 and 2 were used for validation of each assay series. The lyophilized controls contained HE4 antigen in a human serum matrix and a non-azide antimicrobial preservative included in the kit. The mean values of control duplicates and the duplicate replicates of calibrators A-F were within the specified ranges provided by the manufacturer for all runs. The total CV in the present analysis was between 3.3 and $8.8 \%$ in the high and low range of HE4 levels, respectively.

Statistical analyses. A validated HE4 threshold for monitoring during follow-up has not been established, and the current study aimed to investigate changes in HE4 and CA125 during follow-up compared to their 'baseline' level at the end of adjuvant chemotherapy treatment. At 3- and 6-month follow-up examinations, the HE4 and CA125 levels were compared to the baseline end-of-treatment (EOT) sample, and patients were divided into groups based on having an increase above or below/equal to 50\%. From this dichotomous classification, a univariate Kaplan-Meier log-rank analysis was performed to assess the association with progression-free survival (PFS).

Multivariate analysis (Cox regression) was conducted for the identification of independent factors predicting PFS.

The study also aimed to investigate whether the EOT sample drawn at the end of chemotherapy (corresponding to the beginning of follow-up) was able to predict disease recurrence at this very early time point. It was decided a priori that the data would be analyzed at a set sensitivity of $90 \%$ since the aim was to investigate whether the markers were sensitive enough to detect relapse from a serum sample taken just before the follow-up. A sensitivity of $90 \%$ for detecting recurrence was achieved when the threshold was $41 \mathrm{pmol} / \mathrm{l}$ for HE 4 , and $1 \mathrm{U} / \mathrm{ml}$ for CA125. The latter, which is the lowest detectable level, was not meaningful in the analysis of specificity, positive predictive value (PPV) or negative predictive value (NPV) for CA125.

For the analysis of HE4 and CA125 thresholds, simple and multiple regression analyses were used. Statistical analyses were performed with NCSS software (version 2007; NCSS, Kaysville, UT, USA; www.ncss.com) and STATA 13.1 (College Station, TX, USA). The Mann-Whitney U test was used for the comparison of medians. $\mathrm{P}<0.05$ was considered to indicate statistically significant differences.

\section{Results}

Patient characteristics. From May 2006 through August 2011, a total of 283 consecutive patients were enrolled in the 
Table I. Patient characteristics $(n=88)$.

\begin{tabular}{|c|c|}
\hline Clinicopathological parameter & Value \\
\hline \multicolumn{2}{|l|}{ Age, years } \\
\hline Median & 64.0 \\
\hline Range & $28-77$ \\
\hline \multicolumn{2}{|l|}{ FIGO stage, n (\%) } \\
\hline $\mathrm{I}$ & $22(25.0)$ \\
\hline II & $7(8.0)$ \\
\hline III & $44(50.0)$ \\
\hline IV & $15(17.0)$ \\
\hline \multicolumn{2}{|l|}{ Grade $^{\mathrm{a}}, \mathrm{n}(\%)$} \\
\hline 1 & $11(16.9)$ \\
\hline 2 & $25(38.5)$ \\
\hline 3 & $29(44.6)$ \\
\hline \multicolumn{2}{|l|}{ Histological type, n (\%) } \\
\hline Serous & $65(73.9)$ \\
\hline Mucinous & $4(4.5)$ \\
\hline Endometrioid & $9(10.2)$ \\
\hline Clear cell & $6(6.8)$ \\
\hline Other $^{\mathrm{b}}$ & $4(4.5)$ \\
\hline \multicolumn{2}{|l|}{ Residual tumor, n (\%) } \\
\hline $0 \mathrm{~cm}$ & $56(63.6)$ \\
\hline$<1 \mathrm{~cm}$ & $11(12.5)$ \\
\hline$\geq 1 \mathrm{~cm}$ & $21(23.9)$ \\
\hline
\end{tabular}

translational research protocol, and all had serum samples drawn during chemotherapy. In July 2008, the protocol was amended and approved for collection of blood samples during follow-up, and 88 patients were identified as having a serum EOT sample and $\geq 2$ sequential samples collected during the follow-up period, according to the inclusion criteria. The median follow-up time for patients still alive $(n=52)$ was 47 months (range, 26-86 months). Of the 88 patients, 55 were diagnosed with recurrence and 38 patients died during follow-up. More than $97.7 \%$ had $\geq 3$ serial serum samples, and $72.7 \%$ had $\geq 4$ serial samples (maximum, 12). The median time between collection of the EOT sample and the first follow-up sample at 3 months was 93 days (31-147 days) and the median time to the sample drawn at 6 -month follow-up was 187.5 days (68-266 days). In total, 547 serum samples were analyzed: 83 EOT samples and 464 samples during the subsequent follow-up period. Patient characteristics are presented in Table I.

Prediction of relapse from EOT samples by CA125, HE4 and combined CA125/HE4 levels. The median CA125 serum level at the end of first-line chemotherapy treatment (prior to the initiation of follow-up) was $4 \mathrm{U} / \mathrm{ml}$ (95\% CI, 1-5 U/ml; range 1-14 U/ml) for patients without relapse and $5 \mathrm{U} / \mathrm{ml}(95 \%$

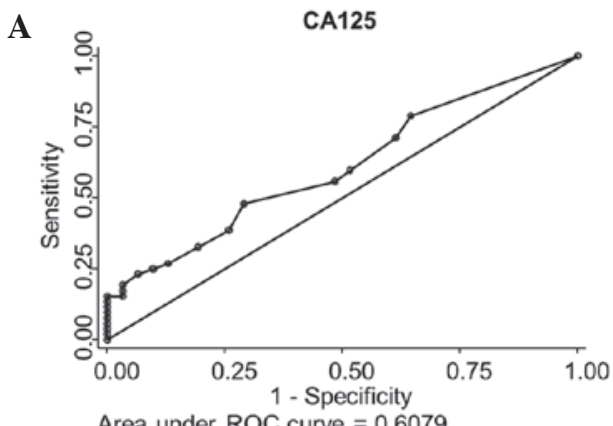

Area under ROC curve $=0.6079$
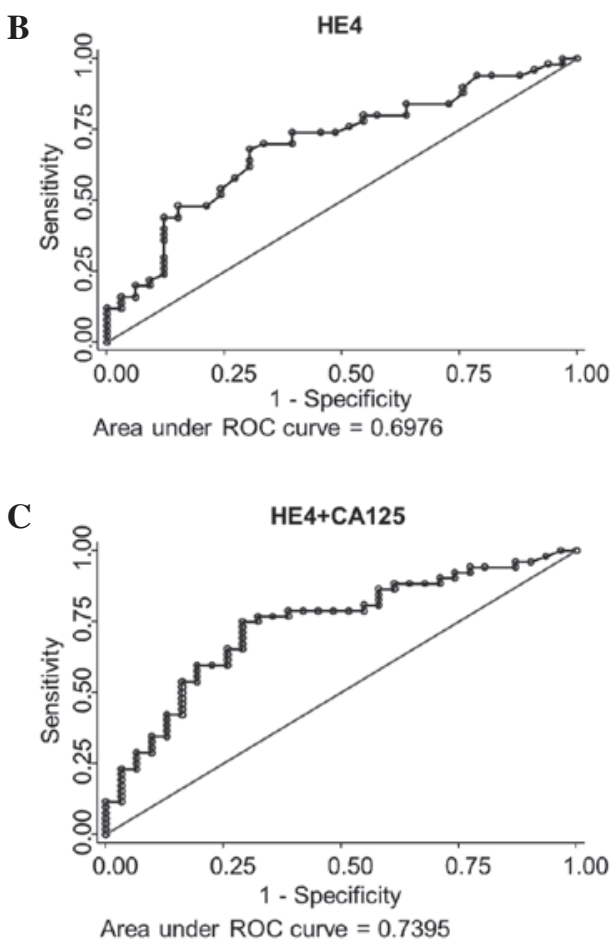

Figure 1. ROC curves for women with ovarian cancer with relapse as the endpoint. Risk stratification was performed using blood sample drawn at the end of first line adjuvant treatment prior to follow-up. (A) CA125, (B) HE4 and (C) combined HE4 and CA125 at the end of treatment for cases (relapse) vs. patients with no relapse during follow-up. ROC, receiver operating characteristic; HE4, human epididymis protein 4; CA125, carbohydrate antigen 125.

CI, 3-7 U/ml; range 1-116 U/ml) for patients with relapse ( $\mathrm{P}=0.0985$, Mann-Whitney U test).

The median HE4 serum level at the end of first-line chemotherapy treatment (prior to the initiation of follow-up) was $51 \mathrm{pmol} / 1$ (95\% CI, 46-59 pmol/1; range, 15-127 pmol/1) for patients without relapse and $67 \mathrm{pmol} / \mathrm{l}(95 \% \mathrm{CI}, 60-81 \mathrm{pmol} / \mathrm{l}$; range, 31-229 pmol/1) for patients with relapse $(\mathrm{P}=0.0013$, Mann-Whitney U test).

Fig. 1 illustrates the receiver operating characteristic curve analysis for samples collected at the end of first-line treatment prior to follow-up (EOT sample), and prediction of recurrence with relapse (PFS) as the endpoint.

HE4 values at the end of first-line treatment classified 70 patients $(84.3 \%)$ as being in the high risk of relapse group, and $13(15.7 \%)$ into the low-risk group, with a sensitivity of $90.0 \%$ (95\% CI, 79.0-96.8\%), a specificity of $25.8 \%$ (95\% CI, 11.9-44.6\%), a PPV of $67.1 \%$ (95\% CI, 54.9-77.9\%) and an NPV of $61.5 \%$ (95\% CI, 31.6-86.1\%) (data not shown). 

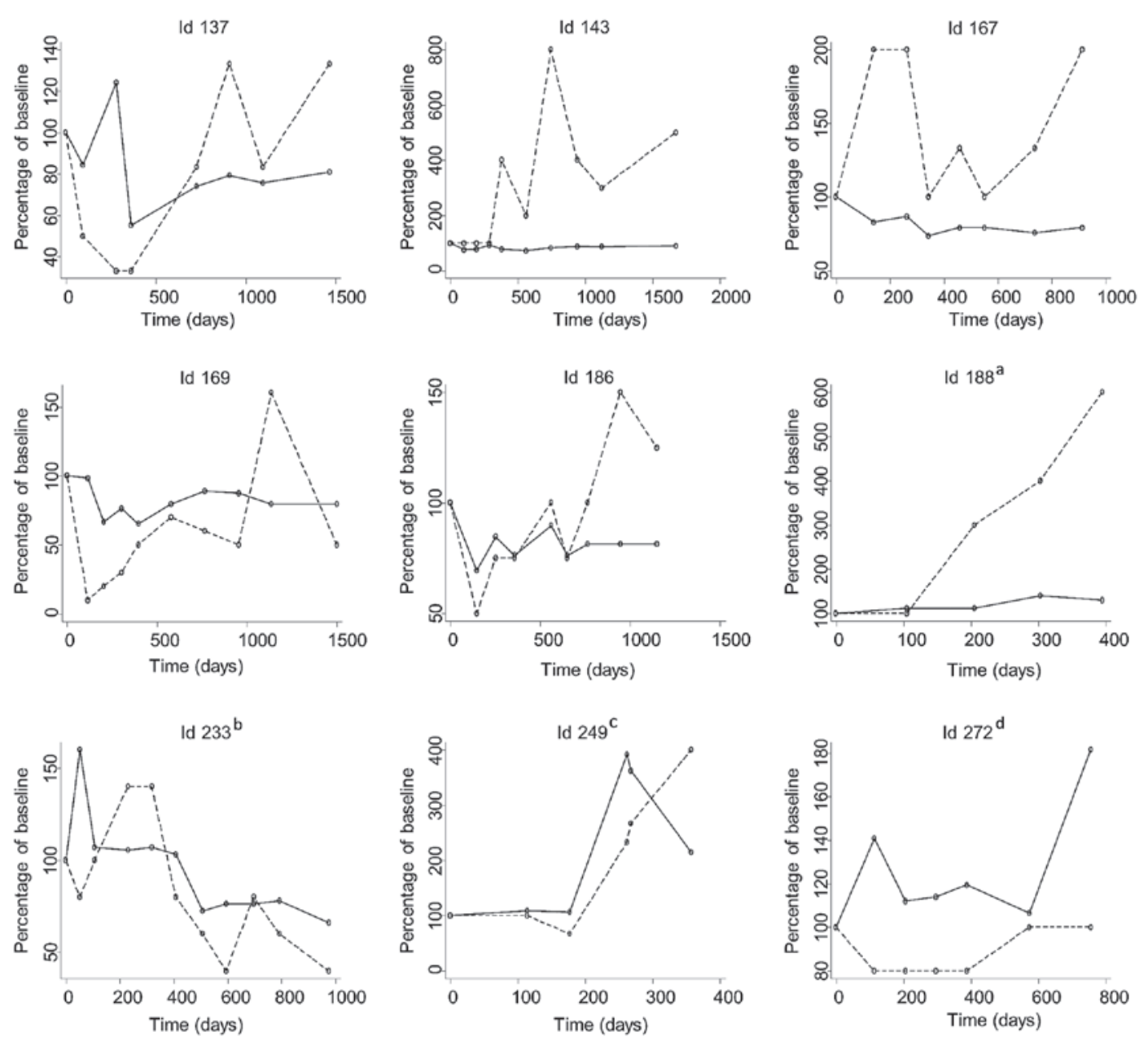

HE4

CA125

Figure 2. Graphs showing the development (in percentage compared to the end-of-treatment sample) of CA125 and HE4 levels during follow-up for selected patients without disease relapse during follow-up. ${ }^{~}$ Id 188 with HE4 levels of 100-150 pmol/1 during follow-up was diagnosed with a metastasizing pancreatic tumor at 16-month follow-up. 'Id 233 only had increased HE4 shortly after the end of chemotherapy treatment, which dropped to within the normal range during follow-up. ' Id 249 was diagnosed with a new primary tumor (a lung adenocarcinoma) at the time of increasing HE4; HE4 increased from 102 to 376 pmol/1 in 3 months just prior to the lung cancer diagnosis. ${ }^{d}$ Id 272 had HE $4>150$ pmol/1 without clinical signs of relapse. HE4, human epididymis protein 4; CA125, carbohydrate antigen 125.

When combining HE4 and CA125, 69 patients (83.1\%) were in the high-risk and $14(16.9 \%)$ in the low-risk group, resulting in a sensitivity of $90.0 \%$ (95\% CI, $79.0-96.8 \%)$, a specificity of $29 \%$ (95\% CI, 14.2-48.0\%), a PPV of $68.1 \%(95 \%$ CI, 55.8-78.8\%) and an NPV of $64.3 \%$ (95\% CI, 35.1-87.2\%) (data not shown).

Prediction of relapse during follow-up. Fig. 2 (patients without relapse) and Fig. 3 (patients with relapse) illustrate selected cases during the follow-up process, in which the first serum sample is the EOT sample and subsequent samples were obtained during the follow-up visits. Analysis of HE4 and CA125 levels at 3 months after EOT (Fig. 4A and B) revealed that an increase of $\geq 50 \%$ (high-risk patients) relative to the level at EOT is associated with a significant worsening of PFS. In particular, a HE4 increase $>50 \%$ was correlated with significant worsening of PFS time [hazard ratio (HR), 2.82; 95\% CI, 0.91-8.79; $\mathrm{P}=0.0052$, log-rank test]. Additionally, increased CA125 ( $>50 \%)$ was associated with poorer PFS time (HR, 1.86; 95\% CI, 0.90-3.80; P=0.0487, log-rank test).
The median PFS was 25.1 months (95\% CI, 17.7-56.8) if HE4 did not increase $>50 \%$ compared to the EOT sample, while it was 11.1 months (95\% CI, 11.0-12.4) for an increase $>50 \%$ at the 3-month follow-up.

For CA125 at 3 months, the median PFS was 28.7 months (95\% CI, 17.4-56.8) if stable, and 13.4 months (95\% CI, $11.5-24.9)$ if increased $>50 \%$.

The impact of increased HE4 became more clear after 6 months of follow-up (HR, 7.71; 95\% CI, 3.03-19.58; P<0.0001, log-rank test) (Fig. 4D and E), with a median PFS time of 56.8 months (95\% CI, 28.7-63.2) if stable, compared with 11.4 months (95\% CI, 10.8-12.1) when HE4 increased $>50 \%$. The corresponding median PFS values were 56.8 months $(95 \%$ CI, 28.7-63.2) vs. 14.2 months (95\% CI, 12.0-7.4) for CA125 (HR, 2.55; 95\% CI, 1.39-4.68, P=0.0011, log-rank test).

Combining the two markers and classifying the patients into a high-risk group if both markers had increased $>50 \%$ revealed similar results: $\mathrm{P}<0.0001$ with a median PFS of 25.1 months (95\% CI, 17.7-56.8) if both markers were stable, and 11.5 months (95\% CI, 9.2-11.6) if both markers increased 

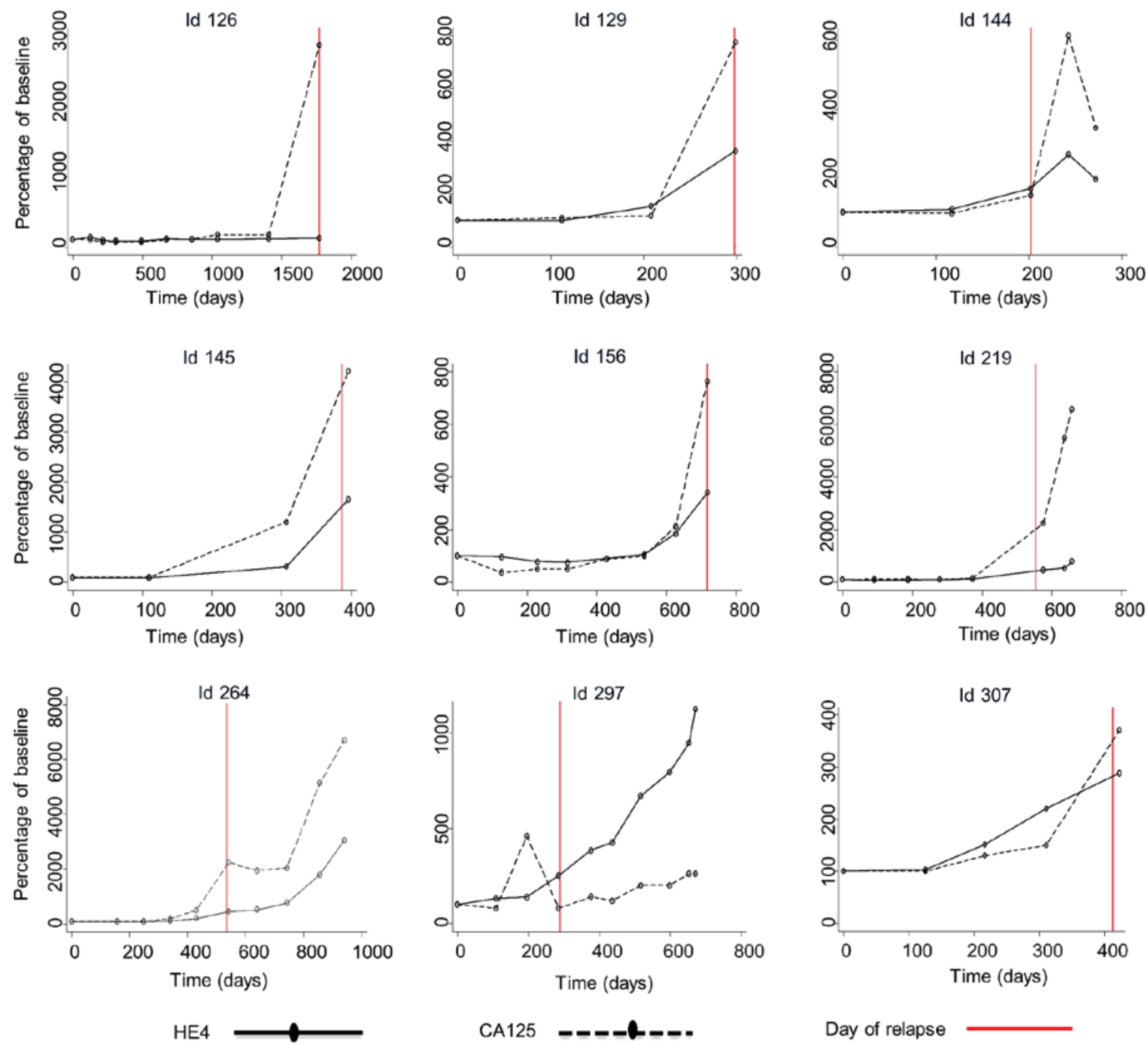

CA125

Day of relapse

Figure 3. Graphs showing the development (in percentage compared to the end-of-treatment sample) of CA125 and HE4 levels during follow-up for selected patients with progressive disease/relapse during follow-up. The vertical line illustrates the time of relapse. HE4, human epididymis protein 4; CA125, carbohydrate antigen 125 .
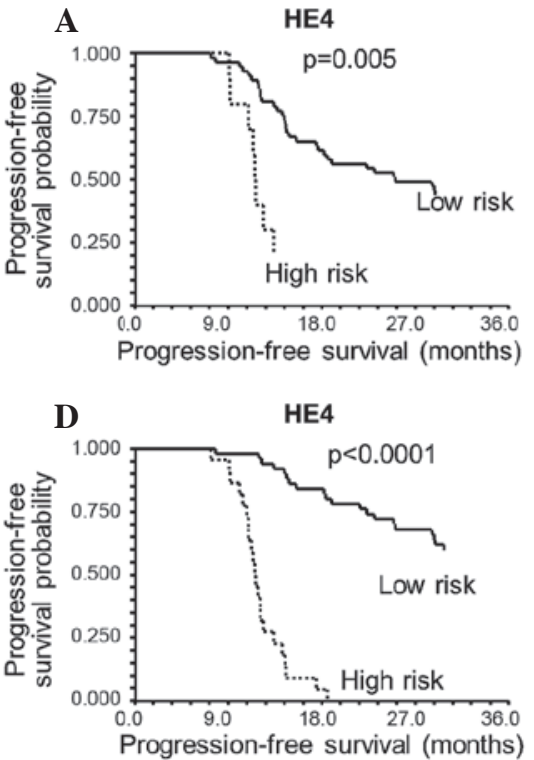

B

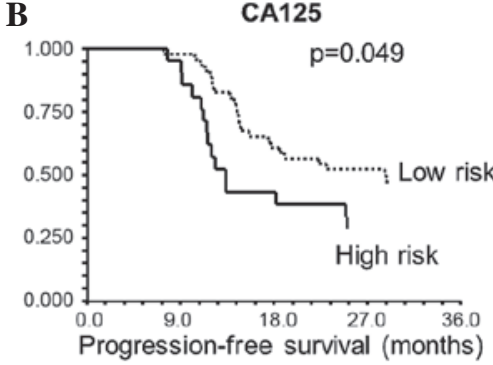

$\mathbf{E}$

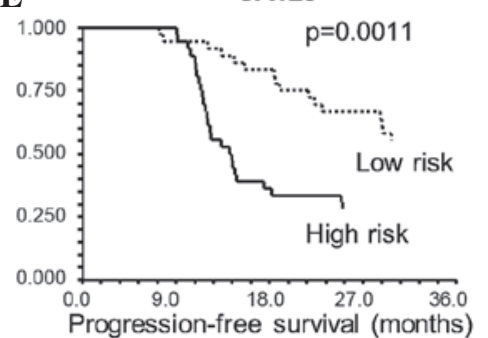

C

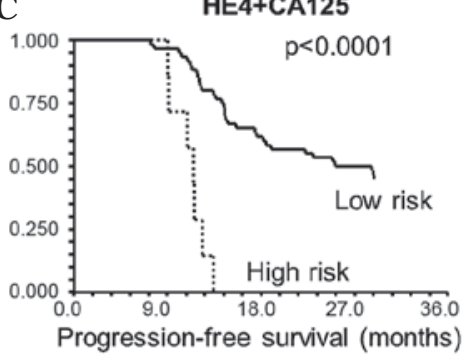

F

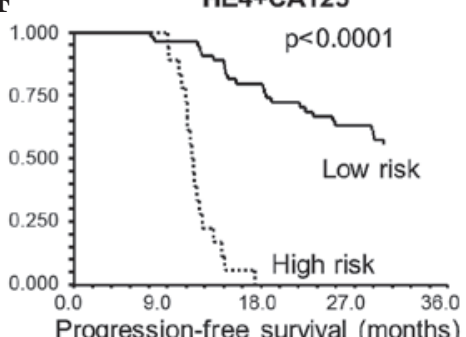

Figure 4. Kaplan-Meier curves for progression-free survival analysis for samples analyzed at (A-C) 3-month and (D-F) 6-month follow-up. Low risk indicates no increase in marker compared to the end-of-treatment sample; high risk indicates an increase in marker of $\geq 50 \%$ compared to the end-of-treatment sample. HE4, human epididymis protein 4; CA125, carbohydrate antigen 125. 
Table II. Multivariate progression-free survival analysis for 3 and 6 months of follow-up.

\begin{tabular}{|c|c|c|c|c|c|c|}
\hline \multirow[b]{2}{*}{ Variable } & \multicolumn{3}{|c|}{ 3-month follow-up } & \multicolumn{3}{|c|}{ 6-month follow-up } \\
\hline & HR & $95 \% \mathrm{CI}$ & P-value & HR & $95 \% \mathrm{CI}$ & P-value \\
\hline Age & 0.97 & $0.94-1.00$ & 0.081 & 0.96 & $0.93-1.00$ & 0.034 \\
\hline \multicolumn{7}{|l|}{ FIGO stage } \\
\hline $\mathrm{I} / \mathrm{II}$ & 1.00 & & Ref & 1.00 & & Ref \\
\hline III/IV & 9.99 & $2.70-36.9$ & $<0.001$ & 5.90 & $1.89-18.4$ & 0.002 \\
\hline \multicolumn{7}{|l|}{ Tumor grade } \\
\hline 1 & 1.00 & & Ref & 1.00 & & Ref \\
\hline 2/3/not graded & 2.54 & $0.86-7.71$ & 0.093 & 1.42 & $0.48-4.15$ & 0.524 \\
\hline \multicolumn{7}{|l|}{ Histology } \\
\hline Serous & 1.00 & & Ref & 1.00 & & Ref \\
\hline Non-serous ${ }^{\mathrm{a}}$ & 1.33 & $0.52-3.40$ & 0.546 & 1.12 & $0.44-2.85$ & 0.816 \\
\hline \multicolumn{7}{|l|}{ Residual tumor } \\
\hline $0 \mathrm{~cm}$ & 1.00 & & Ref & 1.00 & & Ref \\
\hline$<1 \mathrm{~cm}$ & 3.88 & $1.50-10.0$ & 0.005 & 2.06 & $0.84-5.02$ & 0.112 \\
\hline$\geq 1 \mathrm{~cm}$ & 2.17 & $0.92-5.11$ & 0.078 & 2.24 & $0.94-5.35$ & 0.069 \\
\hline \multicolumn{7}{|l|}{ HE4 } \\
\hline Below cut-off & 1.00 & & Ref & 1.00 & & Ref \\
\hline Above cut-off & 1.31 & $0.46-3.72$ & 0.612 & 8.28 & $3.28-20.9$ & $<0.0001$ \\
\hline \multicolumn{7}{|l|}{ CA125 } \\
\hline Below cut-off & 1.00 & & Ref & 1.00 & & Ref \\
\hline Above cut-off & 1.29 & $0.60-2.76$ & 0.513 & 1.45 & $0.67-3.17$ & 0.348 \\
\hline
\end{tabular}

${ }^{a}$ Mucinous, endometrioid, clear cell and other. FIGO stage was divided into stage I/II vs. stage III/IV, grade was divided into grade 1 vs. grade 2/3/not graded, and histology into serous vs. non-serous to avoid too many parameters being entered into the Cox model in relation to the number of events. FIGO, International Federation of Gynecology and Obstetrics; HE4, human epididymis protein 4; CA125, carbohydrate antigen 125; HR, hazard ratio; CI, confidence interval; Ref, reference.

$\geq 50 \%$ at 3 months (Fig. 4C). However, this group of high-risk patients was small at the 3 -month follow-up $(n=7)$. At 6 months, the combination of both markers also predicted PFS $\mathrm{P}<0.0001$ (Fig. 4F), with a median PFS of 56.8 months $(95 \%$ CI, 28.7-63.2) if both markers were stable, and 11.4 months (95\% CI, 10.9-11.9) if both markers increased $\geq 50 \%$.

On multivariate analysis, CA125 was non-significant at 3- and 6-month follow-up, while HE4 was highly significant at 6-month follow-up, with an HR of 8.23 (95\% CI, 3.28-20.9; $\mathrm{P}<0.0001$, Cox regression) (Table II). For the combination of HE4 and CA125 on multivariate analysis, there were too few high-risk patients $(n=7)$ with both markers positive at 3 months for the analysis to be conducted; whereas the HR at 6 months was 7.43 (95\% CI, 2.92-18.9; $\mathrm{P}<0.0001$ ) (data not shown) when both biomarkers increased $\geq 50 \%$.

\section{Discussion}

In June 2008, the HE4 EIA kit (Fujirebio Diagnostics AB) was approved by the FDA to monitor recurrence or progressive disease in patients with epithelial ovarian cancer. In September 2011, the FDA approved marketing of the HE4 test (Fujirebio Diagnostics, Malvern, Pennsylvania) in combination with the CA125 test in the Risk of Ovarian Malignancy
Algorithm (ROMA ${ }^{\mathrm{TM}}$ ) as a diagnostic tool for determining the likelihood of malignancy at the time of surgery in women presenting with an ovarian adnexal mass. ROMA ${ }^{\mathrm{TM}}$ is a qualitative serum test that combines the results of HE4 EIA, ARCHITECT CA125 II ${ }^{\mathrm{TM}}$ (not the same CA125 test used by the present study) and menopausal status into a numerical value and classifies women as being at low- or high-risk for malignant disease. This risk is given as an adjunct to the two test results for CA125 and HE4.

Another possible application of HE4 is during follow-up, which has only been sparsely investigated (26-31). A simple approach is to analyze the marker at the EOT in an effort to identify a group of patients at high risk of early recurrence, an important aspect prompting for further investigation. The results presented herein indicate that analyzing HE4 at this stage is insufficient for reliable classification with regard to the risk of recurrence.

In the current study, measurements of HE4 at the 3- and 6-month follow-ups demonstrated a significant difference in PFS compared with CA125, with considerably higher hazard ratios for HE4. Combination of the two markers, with classification of the patients into a high-risk group if both markers increased $\geq 50 \%$, did not provide additional value. By reviewing the Kaplan-Meier curves, it appears that HE4 
levels are responsible for delineating the large difference in PFS. This was also found to be true on multivariate analysis: CA125 proved not to be significant at 3- or 6-month follow-up, whereas HE4 was highly significant at 6-month follow-up with an HR of 8.23. Similarly, combining HE4 and CA125 in the multivariate analysis, HE4 was revealed to be the important marker, while CA125 appeared not to complement the prognostic value of HE4 during follow-up.

A study by Havrilesky et al (26) monitored 27 patients with advanced ovarian cancer subsequent to chemotherapy and evaluated a biomarker panel of which HE4 was one. All 27 patients experienced recurrence following initial response to treatment. The sensitivity for predicting recurrence was $100 \%$ for the biomarker panel and $96 \%$ for CA125. In 15 patients $(56 \%), \geq 1$ panel biomarkers were elevated earlier (range, 6-69 weeks) than CA125 and prior to other clinical evidence of recurrence. However, a drawback of this study is the lack of a control group to enable comparison of marker behavior during follow-up in patients with and without recurrence. The current study also demonstrated that, compared to CA125, more patients had elevated HE4 at relapse (or during follow-up), but elevated HE4 was also present in the control group of patients with no clinical detection of relapse.

A study by Plotti et al (27) investigated serum CA125 and HE4 levels in 34 patients with radiological suspicion of recurrence and in 34 patients with benign ovarian tumors. The CA125 sensitivity and specificity for detecting recurrent ovarian cancer were 35.29 and $58.82 \%$, respectively. The HE4 sensitivity values were 73.53 and $26.47 \%$ when using 70 and $150 \mathrm{pmol} / 1$ cut-offs, respectively. HE4 specificity was $100 \%$ (all patients in the ovarian cancer group had relapse of ovarian cancer). When combining CA125 and HE4 at a cut-off of $70 \mathrm{pmol} / \mathrm{l}$, the sensitivity in detecting recurrent ovarian cancer was $76.47 \%$ with a specificity of $100 \%$. It is difficult to compare these results with the current study, since Plotti et al used a patient group with benign tumors for comparison and therefore likely achieved a higher specificity compared to the current results, for which the control group was ovarian cancer patients without recurrence during follow-up.

A relatively recent study by Manganaro et al (28) investigated three consecutive serum samples drawn at 3-month intervals from 21 patients with advanced ovarian cancer. In the 9 patients with relapse, an increase in HE4 (>150 pmol/l) was noted in 22,78 and $89 \%$ of the patients according to the time interval from surgery (1-3 months from surgery, 4-6 months and 7-10 months from surgery, respectively). Only $44 \%$ of the patients with relapse showed CA125 levels $>35 \mathrm{U} / \mathrm{ml}$ at 7-10 months from surgery. None of the 12 patients with stable disease had HE4 levels $>150 \mathrm{pmol} / \mathrm{l}$, whereas 4 patients had CA125 levels $>35 \mathrm{U} / \mathrm{ml}$. These results are in agreement with those of the current study, wherein the predictive value of HE4 also increased with the time interval.

Only a few other studies, which have included $\leq 20$ patients, have investigated HE4 during follow-up, with similar results (29-31).

All of the previous studies are substantially smaller than the current study and do not have a control group of a reasonable number of patients without clinical recurrence/progressive disease. For certain of the studies, it is not clear when follow-up blood samples were taken, and some samples appear to be drawn during the chemotherapy. The material used in the present study was prospectively collected and retrospectively analyzed as part of a prospective marker protocol, and blood tests were recorded regularly during follow-up. The patients were not retrospectively identified, since their recurrence was already known, and a group of patients with no clinical relapse were available for marker comparison. We have previously published the dynamics of HE4 and CA125 during chemotherapy, and therefore this was not in the scope of the present study (32).

The largest of the previously described studies is the study by Plotti et al (27). As a criterion for inclusion, these patients had radiological signs of relapse at the time of serum sample collection. Therefore, the sample was drawn at time of diagnosis for recurrent disease and not as part of a follow-up study. The $100 \%$ specificity is obvious when all included patients had recurrent disease at sample collection. Furthermore, no comparisons with patients without relapse were performed, and only comparisons with a control group $~ 30$ years younger than an average ovarian cancer cohort, and in which every individual had a benign ovarian tumor.

Early treatment of recurrence may not lead to an improved overall survival time based on therapies available at present (33). Nevertheless, the majority of ovarian cancer patients with advanced disease at diagnosis will relapse after primary treatment, with a dismal prognosis (34). Therefore, investigation of the level of serum markers in patients under monitoring may be essential in distinguishing patients at risk of relapse from those with less aggressive disease. HE4 appears to be a sensitive and specific marker for the detection of recurrence and, in some cases, has the potential to detect recurrence in patients in whom CA125 is negative. However, based on the present results, investigating consecutive blood samples in comparison to a single blood test drawn at the time of diagnosis is not as simple as previously described in the literature. The picture also looks different when marker levels are compared with those of ovarian cancer patients without known relapse, instead of with a group of healthy women. What is clear from the current study and other studies is that there will be cases in which CA125 is not workable and in which HE4 may be a better marker of recurrence.

In conclusion, the results presented here indicate that an early increase of $>50 \%$ of HE4 in the follow-up period relative to the EOT suggests a high risk of recurrence. This opens the perspective of early treatment. However, the results call for confirmation in a larger number of patient samples.

\section{Acknowledgements}

The author appreciate the skilled work by laboratory technicians Camilla Davidsen and Sara Egsgaard, who handled all the blood samples and performed the CA125 and HE4 analyses. The authors also wish to thank the study nurse at Vejle Hospital, Yvette Schandorf Sørensen; and the study nurses at Aalborg Hospital, Kirsten Lambæk and Janni Møldrup, who helped to keep track of the included patients and patient data.

The study was supported by grants from Vejle Hospital and The Cancer Foundation. The present paper was also supported by Fujirebio Diagnostics AB, who kindly provided the kits for the CA125 and HE4 immunoassays. 


\section{References}

1. Siegel R, Naishadham MA and Jemal A. Cancer statistics 2012. CA Cancer J Clin 62: 10-29, 2012.

2. Liu PY, Alberts DS, Monk BJ, Brady M, Moon J and Markman M: An early signal of CA-125 progression for ovarian cancer patients receiving maintenance treatment after complete clinical response to primary therapy. J Clin Oncol 25: 3615-3620, 2007.

3. Vergote I, Rustin GJ, Eisenhauer EA, Kristensen GB Pujade-Lauraine E, Parmar MK, Friedlander M, Jakobsen A and Vermorken JB: Re: New guidelines to evaluate the response to treatment in solid tumors [ovarian cancer]. Gynecologic Cancer Intergroup. J Natl Cancer Inst 92: 1534-1535, 2000.

4. Rustin GJ, Nelstrop AE, Tuxen MK and Lambert HE: Defining progression of ovarian carcinoma during follow-up according to CA 125: A north thames ovary group study. Ann Oncol 7: 361-364, 1996.

5. Tuxen MK, Sölétormos G and Dombernowsky P: Serum tumor marker CA 125 for monitoring ovarian cancer during follow-up. Scand J Clin Lab Invest 62: 177-188, 2002.

6. Rustin GJ, van der Burg ME, Griffin CL, Guthrie D, Lamont A, Jayson GC, Kristensen G, Mediola C, Coens C, Qian W, et al: Early versus delayed treatment of relapsed ovarian cancer (MRC OV05/EORTC 55955): A randomised trial. Lancet 376 : $1155-1163,2010$

7. Harter P: DESKTOP III (AGO-OVAR OP.4): A randomized trial evaluating cytoreductive surgery in patients with platinum-sensitive recurrent ovarian cancer. http://www.gcig.igcs. org/ClinicalTrials.html.

8. Fleming ND, Cass I, Walsh CS, Karlan BY and Li AJ: CA125 surveillance increases optimal resectability at secondary cytoreductive surgery for recurrent epithelial ovarian cancer. Gynecol Oncol 121: 249-252, 2011.

9. Einhorn N, Bast RC Jr, Knapp RC, Tjernberg B and Zurawski VR Jr: Preoperative evaluation of serum CA 125 levels in patients with primary epithelial ovarian cancer. Obstet Gynecol 67: 414-416, 1986.

10. Clauss A, Lilja $\mathrm{H}$ and Lundwall A: A locus on human chromosome 20 contains several genes expressing protease inhibitor domains with homology to whey acidic protein. Biochem J 368 233-242, 2002.

11. Bouchard D, Morisset D, Bourbonnais Y and Tremblay GM: Proteins with whey-acidic-protein motifs and cancer. Lancet Oncol 7: 167-174, 2006.

12. Bouchard D, Morisset D, Bourbonnais Y and Tremblay GM: Proteins with whey-acidic-protein motifs and cancer. Lancet Oncol 7: 167-174, 2006.

13. Galgano MT, Hampton GM and Frierson HF Jr: Comprehensive analysis of HE4 expression in normal and malignant human tissues. Mod Pathol 19: 847-853, 2006.

14. Drapkin R, von Horsten HH, Lin Y, Mok SC, Crum CP, Welch WR and Hecht JL: Human epididymis protein 4 (HE4) is a secreted glycoprotein that is overexpressed by serous and endometrioid ovarian carcinomas. Cancer Res 65: 2162-2169, 2005.

15. Hellström I, Raycraft J, Hayden-Ledbetter M, Ledbetter JA, Schummer M, McIntosh M, Drescher C, Urban N and Hellström KE: The HE4 (WFDC2) protein is a biomarker for ovarian carcinoma. Cancer Res 63: 3695-3700, 2003.

16. Hellstrom I and Hellstrom KE: SMRP and HE4 as biomarkers for ovarian carcinoma when used alone and in combination with CA125 and/or each other. Adv Exp Med Biol 622: $15-21,2008$

17. Hellstrom I and Hellstrom KE: fTwo novel biomarkers, mesothelin and HE4, for diagnosis of ovarian carcinoma. Expert Opin Med Diagn 5: 227-240, 2011.
18. Montagnana M, Lippi G, Ruzzenente O, Bresciani V, Danese E, Scevarolli S, Salvagno GL, Giudici S, Franchi M and Guidi GC: The utility of serum human epididymis protein 4 (HE4) in patients with a pelvic mass. J Clin Lab Anal 23: 331-335, 2009.

19. Moore RG, Brown AK, Miller MC, Skates S, Allard WJ, Verch T, Steinhoff M, Messerlian G, DiSilvestro P, Granai CO and Bast RC Jr: The use of multiple novel tumor biomarkers for the detection of ovarian carcinoma in patients with a pelvic mass. Gynecol Oncol 108: 402-408, 2008

20. Moore RG, McMeekin DS, Brown AK, DiSilvestro P, Miller MC, Allard WJ, Gajewski W, Kurman R, Bast RC Jr and Skates SJ: A novel multiple marker bioassay utilizing HE4 and CA125 for the prediction of ovarian cancer in patients with a pelvic mass. Gynecol Oncol 112: 40-46, 2009.

21. Steffensen KD, Waldstrøm M, Brandslund I and Jakobsen A: Prognostic impact of prechemotherapy serum levels of HER2, CA125, and HE4 in ovarian cancer patients. Int J Gynecol Cancer 21: 1040-1047, 2011.

22. Steffensen KD, Waldstrøm M and Jakobsen A: The relationship of platinum resistance and ERCC1 protein expression in epithelial ovarian cancer. Int J Gynecol Cancer 19: 820-825, 2009.

23. World Medical Association Declaration of Helsinki: ethical principles for medical research involving human subjects. JAMA 310: 2191-2194, 2013.

24. Rustin GJ: Use of CA-125 to assess response to new agents in ovarian cancer trials. J Clin Oncol 21 (Suppl 10): S187-S193, 2003.

25. Rustin GJ, Quinn M, Thigpen T, du Bois A, Pujade-Lauraine E, Jakobsen A, Eisenhauer E, Sagae S, Greven K, Vergote I, et al: Re: New guidelines to evaluate the response to treatment in solid tumors (ovarian cancer). J Natl Cancer Inst 96: 487-488, 2004.

26. Havrilesky LJ, Whitehead CM, Rubatt JM, Cheek RL, Groelke J, He Q, Malinowski DP, Fischer TJ and Berchuck A: Evaluation of biomarker panels for early stage ovarian cancer detection and monitoring for disease recurrence. Gynecol Oncol 110: 374-382, 2008.

27. Plotti F, Capriglione S, Terranova C, Montera R, Aloisi A, Damiani P, Muzii L, Scaletta G, Benedetti-Panici P and Angioli R: Does HE4 have a role as biomarker in the recurrence of ovarian cancer? Tumour Biol 33: 2117-2123, 2012.

28. Manganaro L, Michienzi S, Vinci V, Falzarano R, Saldari M, Granato T, Viggiani V, Frati L and Anastasi E: Serum HE4 levels combined with CE CT imaging improve the management of monitoring women affected by epithelial ovarian cancer. Oncol Rep 30: 2481-2487, 2013.

29. Anastasi E, Marchei GG, Viggiani V, Gennarini G, Frati L and Reale MG: HE4: A new potential early biomarker for the recurrence of ovarian cancer. Tumour Biol 31: 113-119, 2010.

30. Schummer M, Drescher C, Forrest R, Gough S, Thorpe J, Hellström I, Hellström KE and Urban N: Evaluation of ovarian cancer remission markers HE4, MMP7 and Mesothelin by comparison to the established marker CA125. Gynecol Oncol 125: 65-69, 2012

31. Granato T, Midulla C, Longo F, Colaprisca B, Frati L and Anastasi E: Role of HE4, CA72.4, and CA125 in monitoring ovarian cancer. Tumour Biol 33: 1335-1339, 2012.

32. Steffensen KD, Waldstrøm M, Brandslund I, Petzold M and Jakobsen A: The prognostic and predictive value of combined HE4 and CA-125 in ovarian cancer patients. Int J Gynecol Cancer 22: 1474-1482, 2012.

33. Rustin GJ, van der Burg ME, Griffin CL, Guthrie D, Lamont A, Jayson GC, Kristensen G, Mediola C, Coens C, Qian W, et al: Early versus delayed treatment of relapsed ovarian cancer (MRC OV05/EORTC 55955): A randomised trial. Lancet 376: 1155-1163, 2010.

34. National Cancer Institute: Cancer statistics: SEER stat fact sheets, ovary cancer. http://seer.cancer.gov/statfacts/html/ovary. html. Accessed April 14, 2016. 